\title{
Hyaluronidase, Ovine
}

National Cancer Institute

\section{Source}

National Cancer Institute. Hyaluronidase, Ovine. NCI Thesaurus. Code C65861.

A highly purified preparation of ovine testicular hyaluronidase with potential

chemoadjuvant activity. Upon subcutaneous administration, hyaluronidase modifies the permeability of connective tissue by hydrolyzing hyaluronic acid. This temporarily decreases interstitial viscosity and allows drugs that are co-injected to spread rapidly through the interstitial space, thereby facilitating absorption and/or distribution of coinjected agents. 